\title{
Syndromic Surveillance of Emergency Department Visits for the 2015 Special Olympics
}

\author{
Emily Kajita*, Monica Z. Luarca, Choiyuk Chiang, Han Wu and Bessie Hwang \\ Los Angeles County Department of Public Health, Los Angeles, CA, USA
}

\section{Objective}

To describe how syndromic surveillance was used to monitor health outcomes in near real-time during the 2015 Special Olympics in Los Angeles County (LAC), California.

\section{Introduction}

LAC hosted the 2015 Special Olympics (SO) which welcomed approximately 6,500 athletes from 165 countries, as well as 30,000 volunteers and 500,000 spectators from July 25 to August 2, 2015. International athletes were not required to show proof of vaccinations and were housed in dormitories for nine days, creating potential for infectious disease outbreaks. In response to these unique public health challenges, we describe how LAC's syndromic surveillance system (SSS), which captures over 65\% of all Emergency Department (ED) visits, was used to detect potential emerging health events congruent with $\mathrm{SO}$ games and pre-game events.

\section{Methods}

The SSS team queried ED visit databases for key terms within chief complaint, triage notes and diagnosis fields during the surveillance period of July 20 to August 7, 2015. In addition to monitoring events of particular interest in the SO setting such as Category A bioterrorism agents, meningitis and measles, one additional query was created to identify SO attendees. In order to increase response rates, we requested that ED staff proactively tag within an SO attendee's chief complaint record the phrase "Special Olympics." Upon request, we worked with hospital staff to pinpoint where in the ED registration software keywords could be entered for capture within the syndromic feed. Line lists were reviewed daily.

For syndromes with common or multifactorial symptoms such as gastrointestinal, rash, neurological, respiratory, heat, syncope and seizure, we performed 120 day trend analyses. We focused the analyses geographically by determining the EDs that served the most patients in the five regions where dormitories and competition venues were located. We calculated signal thresholds using modified cumulative sum algorithms.

All surveillance results were created using SAS and summarized in a customized SO syndromic surveillance report generated in Microsoft Word, consisting of manually reported short synopses of case detection results as well as graphs and signal summary tables that automatically updated as source files were output by SAS.

\section{Results}

The SO syndromic surveillance report was created daily for a three week time period (one week before, during and after the SO). No ED visits due to measles or Category A bioterrorism agents were detected during the surveillance period. There were 37 visits with mention of meningitis, however they were unrelated to the SO.

Four hospitals were able to provide screenshots and test patient validation, from which $16 \mathrm{SO}$ labeled ED patients were detected. Of these, two had symptoms consistent with possible gastrointestinal illness, and the rest had physical injury or symptoms such as syncope, seizure and chest pain with nonspecific causes.

Of syndromes that we monitored for regional trends, there were cumulatively eleven signals over three regions. None were sustained for over one day, and counts were close to thresholds and comparable to intermittent peaks in the past 120 days.

\section{Conclusions}

Although the SSS did not detect increases in any syndromes routinely monitored nor in overall ED visits per hospital during the surveillance period, the customized report was a useful tool for summarizing surveillance results for multi-day special events.

Relatively low response rates for SO tagged ED visits may be explained by patients visiting non-syndromic participating EDs, or athletes receiving medical care through SO organized poly clinics and medical stations. Hospitals may also have had experienced barriers to tagging ED records such as the lack of free text based chief complaint data fields or not having enough implementation time in the approximately two weeks given. Others may have tagged patient records but used data fields not captured by the syndromic feed. In the future, we would select fewer but strategically important hospitals to enable more time with each ED to determine eligibility, validate test patients and provide instruction specific to their systems. In the case of the SO, this would have meant soliciting only hospitals closest to the competition venues and dormitories. Although time consuming to implement, proactive tagging of keywords will increase the capture of data specific to special event monitoring.

\section{Keywords}

emergency department; mass gathering; case detection; trend analysis

\section{Acknowledgments}

Many thanks to ACDC's Hospital Outreach Unit nurses for their assistance with hospital outreach in this project and beyond.

\section{*Emily Kajita}

E-mail: ekajita@ph.lacounty.gov 